\title{
Enhancing the Network Life Using Reliable Energy Efficient Routing in Wireless Sensor Networks
}

\author{
Janhavi V \\ Department of Computer science and Engineering \\ Vidya Vardhaka College of Engineering \\ Mysore, India.
}

\author{
A B Ranjendra \\ Department of Computer science and Engineering \\ Vidya Vardhaka College of Engineering \\ Mysore, India.
}

\begin{abstract}
Energy is one of the main network resources that is used in mobile devices. We proposed an energy efficient routing techniques called reliable minimum energy cost routing (RMECR) and comparing with reliable minimum energy routing (RMER) which are used by the nodes in the most efficient manner. RMER and RMECR works on the basis of balancing the energy of the nodes so that the nodes with maximum energy can do the work and the nodes with less than certain threshold energy required to do a certain amount of work will not do any work. Thereby we try to raise the network reliability and lifetime of communication in a network of mobile devices. The proposed RMECR protocol is also used to decrease the cost of communication in a wireless networking environment.
\end{abstract}

Keywords - RMECR protocol; wireless network; mobile devices; reliability; prolonged lifetime

\section{INTRODUCTION}

Wireless sensor network generally consists of spatially distributed autonomous wireless devices which consist of sensors to monitor physical and environmental conditions. Wireless devices are usually mobile devices as they can communicate easily while moving from one place to another. These mobile devices are vastly used in various important applications such as military applications, agriculture, fire detection etc.

Every mobile node in a network is associated with a certain amount of energy. These energy depends on capacity of the battery that is correlate with every node. Each mobile node in the wireless network environment will do some work in transferring the information form source to destination node thereby loses some bulk of energy. The remaining energy(power) of the nodes wises do some work in the network environment is called as residual energy. This residual energy is very essential factor to every mobile node in the wireless environment because it gives how much more work a mobile node can do.

As use of WSNs are increasing day by day providing reliability in transmission is also becoming an important factor. One such routing that can provide reliability to mobile devices are energy effectual routing techniques. Energy efficient routing is one of the main mechanisms possible to decrease the cost of communication in wireless networking environment as there are no secured connections between nodes.

The energy efficient routing technique such as RMER (Reliable Minimum Energy Routing) and RMECR (Reliable Minimum Energy Cost Routing) uses energy efficient paths to propagate through the nodes. It is basically based on the idea of Providing authenticated of links by reducing retransmission. For an instance, De Couto et al. [1] Introduced the represented of expected transmission count (ETX) this is to find secure routes that consist of paths requiring less number of re-transmissions for lost packet recovery.

\section{RELATED WORK}

Energy is an important resource of all wireless sensor nodes in a network. Without the residual energy sensor nodes in WSN switch to off state and stops their working irrespective of the ongoing process, it is used in a proper way to accomplish tasks efficiently in every node in WSN. Hence, using a RMER protocol was proposed by Javad, Venkatesha and Ignas for Energy efficient routing in Wireless Ad-Hoc Network [1].

For efficient communication the distance information of every node is captured in a Neighbor table and it is very helpful during multipath and long range communication network fields [10][6]. For fast access between sender and receiver node communication can achieved by using efficient routing implementation among the sender and receiver [2][4]. Dijkstra's algorithm, it exhibits shortest path communication among the multi paths in network topology adds an extra fast mechanism for transmission [6]. Packet transmission is crucial point where the attackers can hack the network and grab the data information easily so security takes major role in packet transmission [5] .The data is modified because it cannot understand if it is took by the attackers in the communication path between the sender and receiver that is possible only when the good encryption techniques are adopted in our project otherwise there is a possibility of varies types of attacks[14] [15] in the network communication, hence encryption plays a major role on sender side after that decryption happens on the receiver side in a usual 
manner, hence every data is necessary to be encrypt when it passes once from the sender side.

Using a proper energy efficient routing and packet encryption techniques enhances the network life in Wireless sensor network [1][2] [3][4]. Our work is based on all these studies we did from various papers and web references and all these theories and algorithms proposed on energy efficient routing protocols and the factors needed to achieve these factors.

\section{METHODOLOGY}

Here we mainly use protocol called as RMECR protocol for routing. This RMECR is an energy efficient protocol and is aware of energy of all node in the network. Studies clear that RMECR is able to find energy-efficient and secure routes similar to RMER, while also continuous the operational lifetime of the network. Here we consider some number of nodes in a wireless network within certain range calculated using the formula

$$
\sqrt{(x 2-x 1)^{2}+\left(y^{2}-y_{1}\right)^{2}}
$$

After this all the nodes within the range will calculate its neighbor nodes and stores this information in a table called as neighbor table. The nodes use this data to send the data to the suitable neighbors. As every node is associated with same amount of energy and using we associate every node with certain amount of cost value using Dijkstras algorithm.

Usually to send the information the destination node gives the sender node the shortest path possible to reach the destination. The problem with this technique is that the shortest path nodes may not have sufficient energy to receive and transfer information so we use energy efficient routing, which takes into consideration the bulk of energy remaining in each node.

In this type of routing before sending data to the nodes it takes into consideration the bulk of energy remaining on every node called as residual energy.

Before sending the information the nodes always consider the bulk of energy remaining in every node, if and only if the nodes have sufficient energy to transfer the information to other nodes, if the path has node which doesn't have sufficient energy to relay the packets then it finds an intermediate path immediately and sends the information through this path. This may take a longer path, but efficiency increases as there is very less amount of risk of break in the communication due to less energy of nodes.

Like this an efficient routing reduces the cost of communication as this routing also calculate in the cost of nodes. So cost of communication also increases with the prolonged network lifetime and reliability of nodes in the work. Suitable techniques such as RSA are also used for encryption and decryption of the message that is to be transferred. Like this the message is transferred with reduced cost and efficiently depending on energy of nodes. This type of routing thereby increases reliability of all node increases and thereby raise the network lifetime of these mobile devices operating on the network.

The system model of our research work is depicted in the Fig. 3.1. This model has been simulated in an event driven network simulator NS2. The results are drawn with the trace file and are validated with different source and destination.

\section{A. Simple Energy Efficient Model}

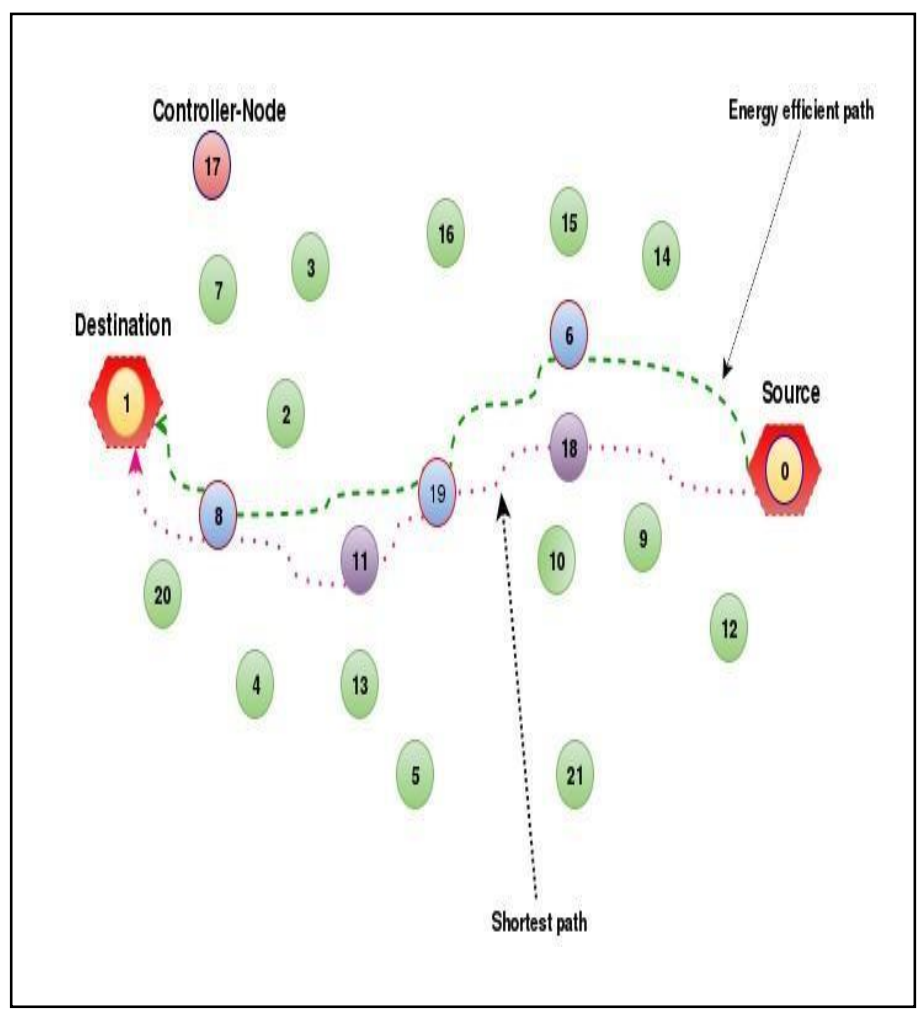

Figure 3.1. Showing the graphical representation of overall energy routing process.

\section{B. Energy Efficient Algorithm}
1.Create requere number of nodes.
2.Initialize all nodes.
3.Calculate distance between nodes.
4.Create neighboring table
If node distance < (certain limit) then
Ignore node
Else then
Include node for communication
5.Apply Dijkstras algorithm. Find shortest path.
6.Check the energy of all node 


If energy of each node< (certain limit) then
Ignore that node from the path
Else then
Choose that node for the routing path
7.Start sending packets.
8.Encryption at the sender side.
9.Transmission in a efficient way.
10.Decryption at the receiver side.
End

\section{Result ANALYSIS}

The energy efficient routing protocol increases throughput, packet delivery ratio, decreases end to end delay when observe to other routing protocols.

The results of our research work are as shown below and is plotted using GNUPLOT.

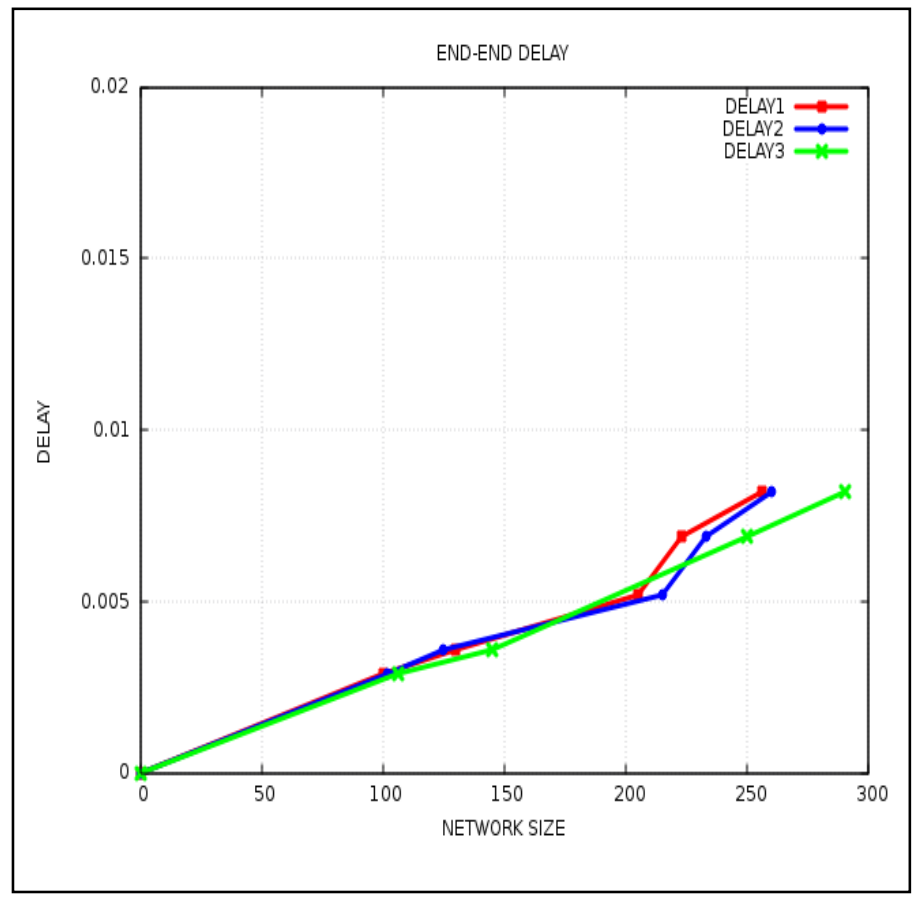

Figure 4.1. Showing end to end delay in energy efficient routing in the network

As seen in the above (Fig 4.1.) end to end delay increases when the network is increases size that is as overhead increases end to end delay also increases but end to end delay is less compared to other routing. Energy efficient routing was increase the Packet Delivery Ratio (PDR)(Fig 4.2). The number of packets received increases drastically by using energy efficient routing as there are no break in the path and no need for retransmission of packets.

In comparative table (Table 1) gives performance ratio on different value of factors in WSN by analyzing this we understand the environment and decide the enhancement on energy efficient routing.

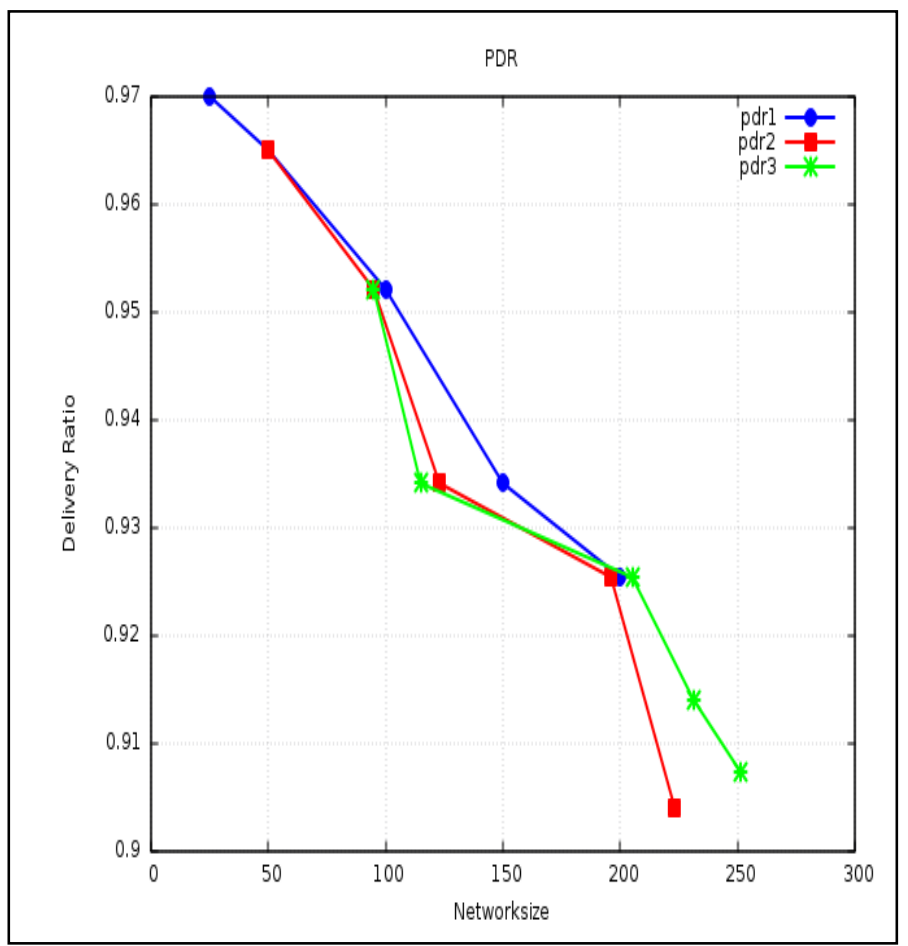

Figure 4.2. Packet Delivery Ratio of mobile devices using RMECR protocol.

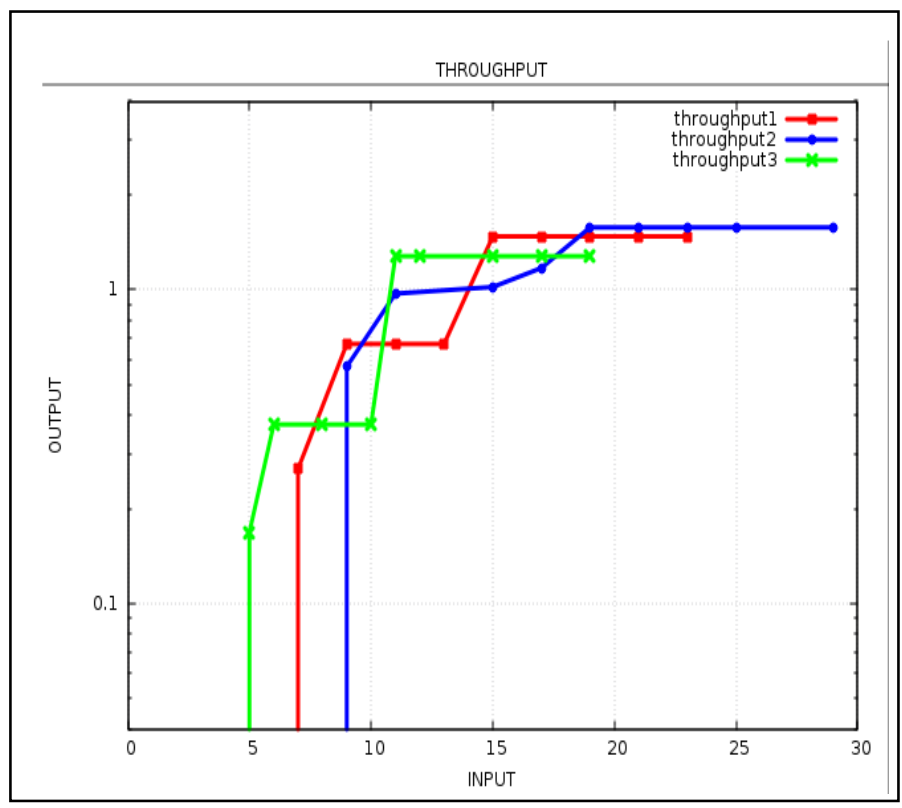

Figure 4.3 Throughput using RMECR protocol 
The above Fig 4.3 shows packet delivery ratio is almost tending to unity but as the packet size increases PDR decreases somewhat as shown in the GNUPLOT. Throughput increases by using RMECR protocol (Fig 4.3). Throughput is very important factor in case of WSN. If the throughput is good only when we can get efficient communication.

TABle 1: Performance MEASUREMENT TABle

\begin{tabular}{|c|c|c|c|c|}
\hline FACTORS & $\begin{array}{l}\text { PACKET } \\
\text { SIZE }\end{array}$ & INTERVAL & $\begin{array}{l}\text { NUMBER } \\
\text { OF NODES }\end{array}$ & $\begin{array}{c}\text { PERFORMANCE } \\
\text { RESULT }\end{array}$ \\
\hline \multirow{4}{*}{$\begin{array}{c}\text { PACKET } \\
\text { DELIVERY RATIO }\end{array}$} & \multicolumn{4}{|r|}{$(\%)$} \\
\hline & 100 & 0.0082 & 39 & 94.25 \\
\hline & 200 & 0.0136 & 39 & 92.72 \\
\hline & 300 & 0.0345 & 39 & 90.65 \\
\hline \multirow{4}{*}{ THROUGHPUT } & \multicolumn{4}{|r|}{ (Kbps) } \\
\hline & 100 & 0.0082 & 39 & 125.18 \\
\hline & 200 & 0.0136 & 39 & 127.61 \\
\hline & 300 & 0.0345 & 39 & 145.00 \\
\hline \multirow{4}{*}{ END-END DELAY } & \multicolumn{4}{|r|}{ (ms) } \\
\hline & 100 & 0.0082 & 39 & 105.05 \\
\hline & 200 & 0.0136 & 39 & 110.63 \\
\hline & 300 & 0.0345 & 39 & 140.43 \\
\hline \multirow{3}{*}{ OVERHEAD } & 100 & 0.0082 & 39 & 7.599 \\
\hline & 200 & 0.0136 & 39 & 8.199 \\
\hline & 300 & 0.0345 & 39 & 8.454 \\
\hline
\end{tabular}

\section{CONCLUSION}

As energy efficiency is one of the most important research topic that is going on in the wireless sensor network. The concept of transmission of energy based on residual energy of the nodes using energy efficient protocols can increase the network lifetime and reliability of all nodes in wireless sensor network. We assert that life time of a node is directly proportional to the overhead of a packets in the nodes. We can adopt this in tactical networks, forest fire detection, agriculture based applications of variety WSN application. RMECR outperforms RMER by considering both optimal cost and residual energy as an important factor in Wireless sensor network.

Further we are intended to enhance the performance of the energy efficient routing protocol by using cluster based routing in WSN.

\section{REFERENCES}

[1] Javad Vazifedhdan, R. Venkatesha Prasad and Ignas Niemergeers, "Energy-Efficient Reliable Routing Considering Residual Energy in Wireless Ad Hoc Networks", IEEE Transaction on mobile computing, Vol 13,NO.2, February 2014

[2] X. M. Zhang, E. B. Wang , J. J. Xia , D. K. Sung," An Estimated Distance-Based Routing Protocol for Mobile Ad hoc Networks", IEEE Transactions on Vehicula, Vol.60 , Issue 7, September 2011

[3] A. F. ShetaB. Solaiman," Evolving clustering algorithms for wireless sensor networks with various radiation patterns to reduce energy consumption", Information Security and Intelligence Control (ISIC), 2012 International Conference IEEE, August 2012

[4] A. S. Alzahrani, M. E. Woodward," End-to-end delay in localized QoS routing ", Communication Systems, 2008. ICCS 2008. 11th IEEE Singapore International Conference,February 2008.

[5] D.S.J. De Couto, D. Aguayo, J. Bicket, and R. Morris, "A HighThroughput Path Metric for Multi-Hop Wireless Routing," Proc. ACM MobiCom, pp. 134-146, 2003.

[6] Akyildiz, I., et al., A survey on sensor networks. IEEE communications magazine, 2002. 40(8): p. 102-114.

www.ijeacs.com 
[7] Javad Vazifedhdan, R. Venkatesha Prasad and Ignas Niemergeers,"Minimum Battery Cost Reliable Routing in Ad Hoc Wireless Networks," Proc.Eighth IEEE Consumer Comm. And Networking Conf,Jan.2011

[8] A.B Mohanoor, S. Radhakrishnan, and V. Sarangan,"Online Energy Aware Routing in Wireless Networks",Ad Hoc Networks, Vol.7,no.5, pp.918-931, july 2009

[9] J-H. Chang and L. Tassiulas, "Maximum Lifetime Routing in Wireless Sensor Networks", IEEE/ACM Trans.Networking, vol 12, no 4, pp.609-619, August.2014

[10] Perkins, C.E, M. Belding-Royer E, "Ad Hoc On-Demand Distance Vector (AODV) Routing “,RFC 3561,2003

[11] Ben-Othman, J. Yahya, "Energy Efficient and QOS Based Routing Protocol for Wireless Sensor Networks", J.Parall. Distrib. Comput. 2010,vol 7, pp. 849-857

[12] D.S.J De Couto, D. Aguayo, J. Bicket and R Moris,” A HighThroughput Path Metric for Multi-Hop Wireless Routing",Proc.ACM Mobicom,pp 134-146,2003

[13] T. Clausen and P. Jacquet, "Optimized Link State Routing Protocol (OSLR)",IETF RFC 3626, www.ietf.org/rfc/rfc3626.txt,2003

[14] Mohammed Arbaz,Nithin H V,Ravindra T R, Shivakirana H K, "Comparative Analysis of Different Types of Attacks in MANET",National conf. on Recent trends, October 2015

[15] HL Gururaj, B Ramesh "An efficient switching TCP (STCP) approach to avoid congestion in ad-hoc networks" Advance Computing Conference (IACC), 2015 IEEE International, Pages 191-195, DOI:10.1109/IADCC.2015.7154696

\section{AUTHORS PROFILE}

Janhavi $\mathbf{V}$ completed her B.E degree in Computer Science \& engineering from Visvesvaraya Technological University, Belgaum ,India. Currently she is working as Associate Professor in the department of Computer science and Engineering at Vidya Vardhaka college of engineering, Mysuru , India. Her area of interest includes Wireless Networks, Mobile Communcation, routing prot networks.

Dr A.B. Rajendra completed his B.E degree in Electronics \& communication from Visvesvaraya Technological University, Belgaum India. Currently he is working as Professor in the department of Computer science and Engineering at Vidya Vardhaka college of engineering, Mysuru ,India. His area of interest include Wireless Networks, Mobile Communication and Information \& Network Security.

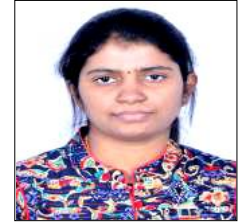
multi-hop wireless

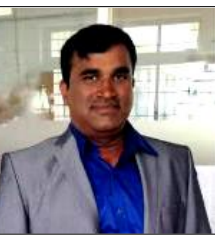

